Judith Galka*, Jolina Haddad und Lea Hartung

\title{
Ein Ort des sozialen Miteinanders: Teilhabeorientierte Bibliotheksarbeit in der Zentral- und Landesbibliothek Berlin
}

https://doi.org/10.1515/bfp-2020-0119

Zusammenfassung: Der Beitrag erkundet praxisgeleitet die wichtige Rolle, die teilhabeorientierte Bibliotheksarbeit für die Entwicklung und Zukunft einer Öffentlichen Bibliothek hat. Er stellt eine Systematik der Partizipation in der Programmgestaltung und den Community-Ansatz anhand von Beispielen vor. Anhand von zwei ausführlichen Beispielen werden Herangehensweisen, Chancen, Konsequenzen und Möglichkeitsgrenzen von teilhabeorientierter Bibliotheksarbeit aufgezeigt.

Schlüsselwörter: Partizipation; Community-Ansatz; Bibliotheks- und Stadtentwicklung

\section{A Place of Social Engagement. The Participatory Approach in the Central and Regional Library Berlin}

\begin{abstract}
Starting from every day experience, the paper explores the role of the participatory approach for the development and the future of public libraries. By explaining a scheme for participation in program development and by illustrating the community-based approach, the consequences, chances, and limits hereof are being discussed.
\end{abstract}

Keywords: Participation; community approach; urban development

\section{Einleitung}

Die Zentral- und Landesbibliothek Berlin (ZLB) versteht sich als Ort für gesellschaftliche Teilhabe und gesellschaftlichen Dialog, als eine Plattform für die Experimentierfreude der Berliner Stadtgesellschaft. Erreichen wollen wir mit unseren vielfältigen Medien-, Programm- und Dienstleistungsangeboten nicht weniger als alle Berliner`innen. Um langfristig als relevante Kultur- und Bildungseinrichtung

*Kontaktperson: Judith Galka, judith.galka@zlb.de

Jolina Haddad, jolina.haddad@zlb.de

Dr. Lea Hartung, lea.hartung@zlb.de
Berlins Bestand zu haben, setzen wir daher auch auf den Austausch, auf das Miteinander, kurz: auf beteiligungsorientierte Bibliotheksarbeit. So ist der vorliegende Text ein Bericht aus der Praxis, die Autorinnen sind selbst in ihrem Arbeitsgebiet mit der Entwicklung und Vermittlung des Themenfelds betraut. Die folgenden Zeilen führen in das Thema ein, erläutern aus der Praxisperspektive mögliche Herangehensweisen, Beispiele und Hürden, vermitteln neben Chancen und Ideen auch Notwendigkeiten und Möglichkeitsgrenzen.

\section{Bibliotheken und partizipative Bibliotheksarbeit}

Was macht eigentlich Bibliotheken für beteiligungsorientierte Vorhaben und Herangehensweisen so interessant? Warum eröffnen Bibliotheken Möglichkeiten der gesellschaftlichen Teilhabe? Der Soziologe Eric Klinenberg sieht in seinem Buch „Palaces for the People“ die Öffentliche Bibliothek, neben anderen ,shared spaces ‘ wie Synagogen, Moscheen, Kirchen, Kindergärten oder Parks, als einen bedeutsamen Bestandteil sozialer Infrastruktur und resilienter Communities, einen Lernort, an dem Menschen die wesentlichen Kompetenzen für gesellschaftliche Teilhabe lernen und damit die Zukunft der Demokratie sichern. Gemeinsam. ${ }^{1}$

Der programmatische Umgang, das Integrieren eines sozialen Miteinanders hin zu teilhabeorientierter Bibliotheksarbeit erfordert neue Strategien und Anpassungen der Organisation, ihrer Strukturen, ihrer Haltung, wie David Lankes schreibt:

\begin{abstract}
„Unser Ziel sollte nicht sein, alles zu sammeln, was unsere Gesellschaft braucht, sondern eine klügere, bewusstere und offenere Gesellschaft in die Welt zu entsenden. [...] Die Menschen, die in Deine Bibliothek kommen, sind keine Konsumenten, die unterhalten oder informiert werden wollen. Sie wollen, dass ihr Leben eine Bedeutung hat. Sie wollen lernen, teilen und teilha-
\end{abstract}

1 Klinenberg (2018). 
ben, die Ingenieure, Klempner, Musiker, Anwälte und viele andere. Diese Menschen bilden den Bestand einer Bibliothek, und der ist viel großartiger als alles, was in den größten Bibliotheken der Welt angesammelt wurde."

Das Thema partizipative Bibliotheksarbeit beschäftigt die ZLB bereits seit einigen Jahren. Seit 2017 wurde aus dem Thema ein neues Geschäftsfeld und als solches in organisatorischer Konsequenz auch ein Programmbereich benannt und in den bibliothekarischen Alltag hinein entwickelt. Partizipation ist in der Geschichte der ZLB zudem stark mit den langen Planungen für einen Bibliotheksneubau verknüpft. Kulminationspunkt bibliothekarischer Programmarbeit und Weiterentwicklung des Neubauvorhabens war der Themenraum „Bibliothek findet Stadt Stadt findet Bibliothek“, der im Jahr 2018 in der ZLB stattfand. Wir beschreiben in unserem ersten Exkurs dieses berlinweite Partizipationsangebot zur Bibliotheksentwicklung genauer, erläutern Zielstellungen, Hürden und Ergebnisse, die sowohl in unsere Programmarbeit als auch in unsere Neubauplanung eingeflossen sind.

Im Zuge unterschiedlicher Anläufe für eine neue Zentralbibliothek für Berlin hat es auch Bestrebungen zum internationalen Austausch gegeben. Bibliotheken und andere Kulturinstitutionen wurden besichtigt, neue Bedarfsprogramme und Herangehensweisen an Bibliotheksarbeit im internationalen Kontext diskutiert und bearbeitet. Mit der Erkenntnis: Die Beteiligung der (Stadt-)Gesellschaft ist dabei für viele der heute von der Fachwelt als besonders erfolgreich anerkannten Bibliotheken zum wesentlichen Grundsatz ihrer Arbeit geworden. So ist das Gebäude des DOKK 1 im dänischen Aarhus für den Austausch und die Angebote von Communities gemacht, der gesamte Entwicklungsprozess der Zentralbibliothek Oodi in Helsinki baut auf eine über zehnjährige Geschichte der Bürger^innenpartizipation auf, die Garaget in Malmö verleiht den Schlüssel zu ihrer Bibliothek regelmäßig an die Communities des umliegenden Bezirks, um dort Veranstaltungen zu machen. Über die Community-Arbeit der ZLB werden wir untenstehend genauer berichten.

Mit hoher Intensität haben wir in der ZLB diesen Prozess vorangetrieben, indem wir einerseits einen eigenen Organisationsbereich geschaffen haben, andererseits war und ist es unser Ziel, die mit beteiligungsorientierter Bibliotheksarbeit verknüpfte gedankliche Haltung in unser gesamtes Kollegium zu tragen. Was sich dabei in den vergangenen Jahren zeigte, war ein Zugleich von Bibliotheksarbeit in ihren zeitgemäßen Kernfunktionen, nämlich der

2 Lankes (2018) 31.
Wissens- und Informationsversorgung der Berliner*innen, bei zeitgleicher Neuentwicklung und Neuverteilung von Aufgaben und Betätigungsfeldern. Die ZLB hat seit 2014 neue Rollen und Funktionen zukünftiger Bibliotheksarbeit ausgelotet und in einem Konzept „Die Bibliothek der $\mathrm{Zu}$ kunft“ auch für die Öffentlichkeit festgehalten. ${ }^{3}$ Wir gehen dabei von drei programmatischen Säulen aus, die Grundlage unserer Arbeit sind:

Erste Säule: Die ZLB als Ort der Informations- und Wissensversorgung, als ein Ort, an dem die Stadt Medien, Informationstechnologie und entsprechende Vermittlungsangebote findet.

Zweite Säule: Die ZLB als (Arbeits-)Ort der Produktivität, als ein Ort, an dem die Stadtgesellschaft Platz zur Arbeit an ihren Themen oder sich selbst findet.

Dritte Säule: Die ZLB als Forum der Stadtgesellschaft, als Plattform der Stadtgesellschaft - als aktivierendes Zentrum mit Gestaltungsspielräumen.

Um diese dritte Säule soll es nun im Folgenden gehen.

\section{Partizipative Bibliotheksarbeit in der ZLB}

Die Vielfalt der Communities macht die besondere Attraktivität der Metropole Berlin aus - und die Öffentlichen Bibliotheken sind Räume, in denen diese Vielfalt gelebt wird. An kaum einem anderen Ort kommen so viele verschiedene Menschen zusammen, die Lust auf Wissen und Erweiterung ihres Horizonts haben - und auf Austausch. Die Mitarbeiter*innen registrieren im Service oft, dass es den Menschen beim Besuch einer Öffentlichen Bibliothek nicht nur darum geht, gezielt ein Medium abzuholen, sondern dass sie oft auch einen großen Wunsch nach Austausch haben. Wir wollen für dieses Austauschbedürfnis ein Marktplatz sein - ein Ort für informierten gesellschaftlichen Dialog, Teilhabe und Mitgestaltung.

Zusätzlich zu den „klassischen“ Bibliotheksressourcen (Medienbestand analog und digital) werden durch die Bibliothek neue Kontakte und Netzwerke zwischen Menschen mit gemeinsamen Interessen oder Austauschbedarf geschaffen, eine Plattform für den Transfer von Information, Wissen und Know-how. Ziel der Plattform-Angebote ist es, die ZLB als Öffentliche Bibliothek auch in Zukunft als

3 ZLB (2018). 
einen relevanten Ort im Leben der Berliner*innen zu verankern, einen Raum, von dem die Berliner`innen sagen: „Das ist mein Ort.“

\section{Form und Struktur - Was bedeutet Partizipation?}

Um verschiedene partizipative Formate $\mathrm{zu}$ unterscheiden, orientieren wir uns im Programmbereich an dem Modell für Partizipation in der Stadtentwicklung von Maria Lüttringhaus $^{4}$ und haben es für den Bibliothekskontext angepasst. ${ }^{5}$ Lüttringhaus definiert zunächst einmal grundlegend Partizipation als die Kommunikation zwischen unterschiedlichen Akteur*innen, die nötig ist, um Ziele zu erreichen. Zu den Beteiligten gehören Zuständige, Betroffene, Interessierte - grundsätzlich kann jede`r einen Partizipationsprozess anstoßen. Partizipation gibt die Gelegenheit, das Wissen über eine Fragestellung zu erweitern.

Die grundlegende Form der Partizipation ist Information: Ohne transparente Kommunikation gibt es keine Teilhabe. Informiert zu sein ist die Grundlage für das Mitdenken, Mitreden und Mitmachen.

Je nachdem, wie die Informationen aufgenommen werden und welche Entscheidungsspielräume bestehen, können weitere Stufen der Partizipation folgen: Konsultation heißt, dass Menschen nach ihrer Meinung, ihren Wünschen und Ideen gefragt werden. Kooperation bedeutet die gemeinsame Suche unterschiedlicher Beteiligter nach einer Lösung für eine Planungsaufgabe oder einen Konflikt. Co-Produktion schließlich bedeutet, dass auch die Umsetzung als Gemeinschaftsaufgabe angegangen wird.

Hierbei setzt sich Partizipation aus zwei aktiven Prozessen zusammen: einerseits die Teilhabegewährung und - im besten Fall - Teilhabestärkung, die von Seiten der Institution erfolgt, andererseits die Teilnahme durch die Bürger`innen. Erst wenn beide Prozesse zusammenkommen, kann man laut Lüttringhaus von Partizipation sprechen.

Dieses Stufenmodell informiert unsere Haltung zum Thema Partizipation. Wir haben die Stufen nicht eins zu eins übernommen, sondern auf unseren spezifischen Bibliothekskontext angepasst und in das Konzept für Partizipation in der Programmgestaltung der ZLB mit einfließen lassen.

4 Lüttringhaus (2000) 44.

5 Hartung et al. (2018).

\section{Partizipation in der Programmgestaltung - Die Bibliothek als Plattform}

Die Bibliothek als Plattform soll es verschiedenen Berliner Communities ermöglichen, in von ihnen (mit-)gestalteten Räumen Gastgeber`innen für Formate ihrer Wahl zu sein. Sandi Hilal fordert Bibliotheken zur Selbstbefragung auf: „Ask yourself: Am I all the time hosting, or am I sometimes the guest at my institution?" ${ }^{\circ}$ Plattform-Formate können in sehr unterschiedlicher Form auftreten. Zwischen der einfachen Zurverfügungstellung von Raum und Infrastruktur seitens der Bibliothek für einzelne Menschen, verfasste Gruppen oder informelle Communities bis hin zu größeren Kooperationsprojekten mit mehreren Partner ${ }^{\star}$ innen ist alles möglich. Wir unterscheiden diese unterschiedlichen Modi im Sinne einer groben Orientierung wie folgt:

Im Modus der Konsultation wird bei der Entwicklung eines Programmformats vorab das Feedback der Zielgruppe bzw. der Community eingeholt und in die Konzeption eingearbeitet. Die Community tritt bei der Veranstaltung nicht notwendigerweise als Gastgeberin mit auf. Ein Beispiel ist die Beratung durch RomaniPhen e.V. Verein von Romnja* und Sintezzi* zur Medienpräsentation für den Konzertdialog mit RomnjaJazz.

Im Rahmen einer Co-Produktion erstreckt sich die $\mathrm{Zu}$ sammenarbeit zwischen ZLB und möglichen Kooperationspartner^innen von der Konzeption bis zur Durchführung in unterschiedlichen Mischungsverhältnissen. Räume und Formate werden gemeinsam entwickelt. Hier sind die Ziele der ZLB und die der Partner`innen beide präsent und die Gastgeber`innen-Rolle wird gemeinsam eingenommen (,co-hosting'). So zum Beispiel bei der Saatgut-Tauschbörse, bei der wir Saatgut-Tausch mit dem Tausch von Wissen darüber in Form von unseren Landwirtschafts-Medien und dem Dialog dazu kombinieren. Im Laufe von zwei Jahren konnten wir für die Saatgut-Tauschbörse verschiedene Kooperationspartner*innen gewinnen (Grüne Bibliothek der Nachbarschaft, Balkon-Beratung, Verein zum Erhalt alter Nutzpflanzensorten, NABU, Weltacker), die die Konzipierung und Durchführung mitgestaltet und bereichert ha-

6 Die Architektin Sandi Hilal hat sich u.a. durch ungewöhnliche Ausbildungsprogramme, kritisches Lernen und demokratische Teilhabeinfrastrukturen in vulnerablen Kontexten einen Namen gemacht. Auf der Next Library Satellite Conference in Berlin 2018 hat sie in ihrer Keynote das Thema Gastfreundschaft adressiert. Vgl. Keynote Sandi Hilal, verfügbar unter https://vimeo.com/290933502. 
ben. Ein anderes Beispiel ist der Presseclub für alle, der als gemeinsame Idee von RiffReporter und ZLB entwickelt und gemeinsam durchgeführt wird. Ein ,Fishbowl-Setting ' lädt jede ${ }^{\star} n$ dazu ein, sich dazuzusetzen und beim aktuellen Thema mitzudiskutieren.

Bei einer Schlüsselübergabe stellt die ZLB einemr Kooperationspartner ${ }^{\star}$ in oder einer Community Raum und Infrastruktur zur Verfügung mit der Geste: „Hier ist der Schlüssel, macht daraus Eure Bibliothek“. Dabei stehen die Ziele der Partner`innen im Vordergrund, die Community ist die Gastgeberin und die ZLB findet sich bei Veranstaltungen in der Rolle des Gasts wieder. Im Hintergrund findet in dieser Form des Plattform-Bietens (,hosting the hosts') eine intensive Zusammenarbeit statt. Ein großes Experimentierfeld für diese Form der Partizipation ist die ZLB-Sonntagsöffnung. ${ }^{7}$ Ein anderes ist unsere Kooperation mit Baynatna, der arabischen Bibliothek, die wir in unserem zweiten Exkurs beschreiben.

\section{Exkurs 1: Teilhabe der Berliner*innen an der Gestaltung der Bibliothek - Der Themenraum „Bibliothek findet Stadt - Stadt findet Bibliothek"}

Durch die Festlegung des Berliner Senats im Juni 2018, den Neubaustandort der ZLB auf das Grundstück an der denkmalgeschützten Amerika-Gedenkbibliothek zu legen, bot sich uns die einmalige Gelegenheit, die Nutzer^innen ganz wortwörtlich an der Gestaltung der zukünftigen ZLB teilhaben zu lassen. „Mitdenken, mitreden, mitmachen“ lautete demnach auch das Motto des Themenraums „Bibliothek findet Stadt - Stadt findet Bibliothek“, in dem es darum ging, wie sich Stadt gemeinsam gestalten lässt und wie man auch als Nicht-Expert`in an der Gestaltung einer neuen ZLB teilhaben kann. Die Medienpräsentation mit Informationen zu Stadtplanung, Partizipationsmöglichkeiten und aktuellen Stadtplanungs- und Bauprojekten, bei denen sich Berliner Initiativen, Interessengruppen und einzelne Bürger`innen in die Zukunft ihrer Stadt einbringen, fungierte hierbei als Hintergrund und Wissensbasis für alle offenen Dialog- und Werkstattformate. $^{8}$

Mit der Leitfrage „Was brauchen Sie für eine Bibliothek, um die Welt zu verändern?“ ist nach den Wünschen der Bürger`innen Berlins für die Bibliothek der Zukunft

7 Leik (2020).

8 Der Themenraum fand unter Projektleitung von Jennifer Borsky statt, vgl. Borsky und Hartung (2019). gefragt worden. Bei zahlreichen Dialogveranstaltungen und Mitmach-Workshops mit insgesamt 45 mitdiskutierenden Expert ${ }^{\star}$ innen und 654 Teilnehmer*innen sowie bei zahlreichen persönlichen Gesprächen mit Bibliotheksbesucher`innen in unseren Publikumsstandorten AmerikaGedenkbibliothek und Berliner Stadtbibliothek gab es viel Austausch. Dabei haben die ZLB-Mitarbeiter`innen viel zugehört - und das Gehörte für die weitere Planung mitgenommen.

Um auch Ideen und Gedanken zum Thema von Menschen miteinzubeziehen, die (noch) nicht zu Bibliotheksbesucher`innen zählen, war eine beauftragte Partizipationsagentur zudem mit einem ZLB-Lastenrad in ganz Berlin auf Tour. Vom Einkaufszentrum im Berliner Stadtteil Lichtenberg über einen Neuköllner Wochenmarkt bis zum Gemeinschaftsgarten auf dem Tempelhofer Feld konnten hierbei ganz unterschiedliche Stimmen zur Leitfrage eingefangen werden.

Durch die kurz vor dem Themenraum bekannt gewordene Entscheidung des Berliner Senats hatte die Frage nach der Wunschbibliothek eine unmittelbare Relevanz für die Menschen aus der Stadt - dementsprechend zahlreich waren die Rückmeldungen. Die ZLB hat insgesamt über 2300 Ideenzettel mit Wünschen und Anregungen gesammelt, die in öffentlichen Arbeitssitzungen sortiert und nach Abschluss des Themenraums in eine einfache interne Datenbank überführt und verschlagwortet wurden. Mithilfe dieser systematischen Erfassung sind die Wünsche und Anregungen der Besucher`innen in die Erstellung des Raum- und Funktionsprogramms für den Architekturwettbewerb für den Neubau eingeflossen. Ziel ist es, im Sinne einer kontinuierlichen Information zum Prozess den Einfluss und das Wirken der Ideen aus der Stadt auch transparent und nachvollziehbar zu machen.

Eine zweite wichtige Erkenntnis aus diesen Erfahrungen für die weitere Ausgestaltung von Partizipation ist es, dass es zum Anbahnen funktionierender Teilhabe einer zielgruppengemäßen Ansprache bedarf. Dabei ist es unerlässlich, den Rahmen und die Art der Mitgestaltung klar und transparent zu formulieren - es muss deutlich sein, wie viele und teilweise auch wie wenige Möglichkeiten bestehen, sich einzubringen. Denn Partizipation hat je nach Vorhaben auch Grenzen.

Eine zweite wichtige Erkenntnis ist die zentrale Rolle, die die Einbeziehung des Personals in partizipative Prozesse spielt. Die Präsenz und Ansprechbarkeit des Bibliothekspersonals war Indiz für Authentizität und fördernder Faktor für die Beteiligung der Besucherinnen - schließlich sind die Mitarbeiter^innen für die Besucherinnen das Gesicht der Bibliotheksarbeit. Im Rahmen einer Ideenwerkstatt wurde von Mitarbeiter^innen das Format der 
„Wünschbar“ entwickelt: Mit dem Angebot von Getränk und Snack wurde im Foyer der Amerika-Gedenkbibliothek das Gespräch mit den Besucher`innen aktiv gesucht: „Was brauchen Sie für eine Bibliothek - was wünschen Sie sich?" Die Antworten wurden auf Ideenzettel festgehalten. Diese „Wünschbar“ mit der Möglichkeit zum persönlichen Dialog zwischen Nutzer^in und Mitarbeiter*in wurde bewusst als Ergänzung zur nicht persönlich betreuten Ideenzettel-Wand im Salon entwickelt.

Ein solches Engagement des Personals setzt wiederum voraus, dass sich die Mitarbeiter`innen informiert, beteiligt, befähigt und berechtigt fühlen, für die Bibliothek zu sprechen. Dies ist eine nicht geringe Herausforderung in einer hierarchischen Organisation. Eine mögliche Antwort darauf ist in der ZLB der sogenannte „Thementag“, eine jährliche Werkstattkonferenz, in der alle Mitarbeiter`innen in unterschiedlichen Formaten am guten Miteinander, an Lösungsstrategien für den Bibliotheksalltag und an der Zukunft der ZLB arbeiten.

\section{Wie kann die Einladung gelingen? Ansätze für die Arbeit mit Communities}

Kehren wir noch einmal zurück zu der Frage der Partizipation im Bibliotheksalltag, in der Gestaltung des Programms. Alle Formen der partizipativen Programmgestaltung (Konsultation, Co-Produktion, Schlüsselübergabe) stehen und fallen mit der aktiven Teilnahme der Besucher^innen. Wie kann die Einladung der Berliner*innen gelingen? Wie wird überhaupt bekannt, dass die ZLB eine Plattform für die Stadtgesellschaft ist? Hier braucht es Impulse, die von der Bibliothek ausgehen, um die Potenziale der Bibliothek als Plattform den aktuellen sowie potenziellen Partner`innen überhaupt erst bewusst zu machen.

Eine Stadtgesellschaft ist nicht monolithisch, sondern heterogen und das lässt sich gut mit dem CommunityBegriff fassen. Wir folgen der Definition von Richard David Lankes von einer Community als einer Gruppe von Menschen, die zusammenkommen, weil sie eine Sache gemeinsam haben: „A community is a set of people who come together around a communality." "Eine Community kann wiederum sehr vielfältig, heterogen, amorph und temporär sein, jedoch gibt es eben die Gemeinsamkeit, aufgrund derer die Menschen zusammenkommen. Um der Heterogenität der Stadtgesellschaft Rechnung zu tragen, müssen einzelne Communities als Zielgruppen identifiziert werden. Dabei spielt das Erkennen der spezifischen Bedürfnisse einer Community eine wichtige Rolle. Ebenso wichtig ist es, sich community-spezifische Diskriminierung und Benachteiligung bewusst $\mathrm{zu}$ machen. Gerade wenn man den Kreis der Besucher*innen erweitern möchte, ist die Ansprache über Communities ein vielversprechender Ansatz. Es geht um die Formulierung der Einladung an die Stadtgesellschaft und ihre Communities, bei uns Programm zu machen. Das Beispiel, das wir für eine Schlüsselübergabe an eine Community genauer beschreiben möchten, ist die Kooperation zwischen der ZLB und „Baynatna. Die arabische Bibliothek“.

\section{Exkurs 2: Schlüsselübergabe am Beispiel von Baynatna mit Nina Simon (OF/BY/FOR ALL)}

Die Community-Arbeit der ZLB versucht, die Bibliothek $\mathrm{zu}$ einem Ort für alle Berliner`innen $\mathrm{zu}$ machen und dabei Barrieren abzubauen, wirklich niederschwellig zu sein und Diskriminierung zu verhindern. Eine der Leitfragen dabei ist: Wie vermeiden wir eine Programm- und Veranstaltungsplanung, die die Bedarfe der Berliner*innen nur antizipiert, ohne sie wirklich zu kennen, und wie schaffen wir es stattdessen, Angebote zu entwickeln, die für die Berliner`innen in ihrer Diversität relevant sind?

Die US-Amerikanerin Nina Simon hat einen Ansatz entwickelt, der Antworten auf diese Fragen geben kann. Nina Simon war Direktorin des Santa Cruz Museum of Art \& History und hat dieses durch eine konsequente Community-Einbindung sowohl in die Personalstruktur als auch in die Programmgestaltung innerhalb weniger Jahre erfolgreich $\mathrm{zu}$ einer lokal, national und international viel beachteten Kultureinrichtung umgestaltet. Ihr Ansatz dient heute weit über die US-amerikanische Museumswelt hinaus Kultureinrichtungen weltweit als Inspiration. Dazu zählen auch immer mehr Bibliotheken.

Ihre Erfolgsformel? Eine Institution muss OF/BY/FOR ALL sein. Das klingt eingängig und bedeutet kurz gesagt, dass eine Institution die Community repräsentieren muss (OF) und ihre Angebote von der Community gestaltet sein müssen (BY), damit sie zu einem Ort für die Community werden kann (FOR ALL). ${ }^{10}$

10 Keynote Nina Simon, Next Library Satellite Conference in Berlin 2018. Verfügbar unter https://vimeo.com/290934027.

9 Lankes (2016) vii. 
Nina Simon hat im Rahmen ihrer Keynote auf der Next Library ${ }^{\circledR}$ Conference Berlin 2018 zwei zentrale Fragen gestellt:

- What are we willing to change to welcome new people?

- What are we willing to change to help new people belong?

Ihre Fragen gehen direkt ans Eingemachte. Denn sie fragen erst gar nicht danach, $o b$ wir innerhalb unserer Institutionen etwas ändern müssen. Nein, sie gehen ganz selbstverständlich davon aus, dass wir etwas ändern müssen, wenn wir ein Ort für unsere Communities sein möchten.

Und hier liegt der besondere Mehrwert von Nina Simons Ansatz. Sie rückt die Community und ihre Bedarfe radikal in unseren Fokus und zwingt uns zu einer selbstkritischen Auseinandersetzung mit unserer eigenen Institution. Bei Nina Simon geht es in allererster Linie darum, der Community zuzuhören, sie kennenzulernen, sie zu verstehen, Vertrauen aufzubauen und unsere Institutionen wo irgend möglich an die Bedarfe der Community anzupassen. Alles ist denkbar und vieles sollte machbar sein.

Es muss nicht immer ein schillerndes Ergebnis sein, dass unsere Anpassung an die Bedarfe unserer Community(ies) symbolisiert. Vielmehr werden Institutionen auch im Kleinen, in den alltäglichen Routinen der teilhabeorientierten Zusammenarbeit mit Communities, auf Strukturen und Routinen innerhalb der eigenen Institution stoßen, die die Zusammenarbeit herausfordern und zur kritischen Selbstreflexion anregen.

Was damit konkret gemeint ist, erläutern wir anhand eines Beispiels aus der ZLB etwas genauer. Seit Anfang 2018 bieten wir dem Projekt Baynatna. Die arabische Bibliothek einen Ort in der ZLB. Seitdem öffnet die arabische Bibliothek immer von Freitag bis Montag ihre Türen im Kleinen Säulensaal der Berliner Stadtbibliothek (dem zweiten Publikumsstandort der ZLB). Das Team von Baynatna betreibt die Bibliothek ehrenamtlich. Neben dem regulären Bibliotheksbetrieb realisiert Baynatna ein buntes und vielseitiges Programm- und Veranstaltungsangebot.

Mit Baynatna haben wir also eine Community im Haus, die für ihre Community Programme entwickelt und anbietet. Das ist fantastisch und wir könnten jetzt hier stehen bleiben. Aber das wäre nicht ganz ehrlich. Denn hinter den Kulissen gibt es auch immer wieder Reibungspunkte. Und diese Reibungspunkte sind aufschlussreich. Warum? Weil sie deutlich machen, dass durch die Zusammenarbeit mit und die Einbindung einer Community eingeübte Strukturen und Routinen innerhalb der eigenen Institution hinterfragt, neu gedacht und wo möglich flexibel angepasst werden müssen, damit die Zusammenarbeit gelingt.
Das berührt beispielsweise die Frage, auf welcher Sprache die Meetings mit Baynatna stattfinden, damit sowohl unsere Kolleg*innen als auch das Team von Baynatna gleichberechtigt teilnehmen können. Finden unsere Treffen auf Deutsch, Englisch, Arabisch oder in allen drei Sprachen statt? Wer fühlt sich in welcher Situation willkommen und wer nicht? Auf wen nehmen wir wann besonders Rücksicht? Oder schaffen wir es, dass sich alle in gleichem Umfang wohl fühlen?

Es berührt auch die Frage, welche Meeting-Routinen wir von einem ehrenamtlichen Team erwarten können. Treffen wir uns monatlich oder vierteljährlich, um Fragen im Rahmen der Kooperation zu besprechen? Können wir ein Treffen zu den regulären Bürozeiten unserer Institution erwarten oder richten wir uns nach dem Kalender des ehrenamtlichen Teams? Und wer macht eigentlich das Agenda-Setting, so dass alle Teilnehmenden das Gefühl haben, auf Augenhöhe zu kommunizieren?

Oder es betrifft die Frage, wann wir als ZLB-Mitarbeiter*innen über geplante Veranstaltungen bei Baynatna informiert werden müssen, sodass wir entsprechend den eingespielten Routinen im Haus allen beteiligten Kolleg*innen bedarfsgerecht Bescheid geben können. Wie schaffen wir es, sowohl die Bedarfe von Baynatna als auch die Bedarfe der Kolleg^innen zu erfüllen? An welchem Punkt sind Zugeständnisse nötig und von wem? Wie passen unsere Routinen mit denen unserer Community zusammen oder eben nicht?

Immer wieder betrifft es auch die Frage, wie viele Abstimmungsschleifen eine Entscheidung innerhalb unserer Institution nehmen muss, sodass relevante Kolleg^innen an der Entscheidung beteiligt werden können. Wie lange dauert ein Prozess innerhalb unserer Institution und wie bekommen wir das in Einklang mit dem Wunsch nach mehr Spontanität und hoher Flexibilität innerhalb der Community, mit der wir kooperieren? Wie erklären wir notwendige Prozesse und an welcher Stelle können wir auf die Community zugehen, um den Bedarf nach mehr Flexibilität zu befriedigen?

Das alles sind zugegebenermaßen scheinbar kleinteilige Fragen. Sie können aber für die zwischenmenschliche Ebene in einer Kooperation und das Gefühl, verstanden und anerkannt zu werden, auf beiden Seiten eine starke Relevanz haben. Dabei berühren viele der Fragen oftmals die ganz allgemeine Frage, wie der Spagat zwischen Verwaltungsstrukturen und zivilgesellschaftlichen Akteur`innen gelingen kann. Einige Strukturen können entsprechend der Bedarfe der Community angepasst werden und andere nicht. Dann braucht es Einfühlungsvermögen und Geduld, um den Sinn bestimmter Vorgaben so zu erklären, dass sie nicht als Gängelung seitens der Institution wahr- 
genommen werden. Und manchmal braucht es auch einfach eine hohe Frustrationstoleranz, wenn der Spagat zwischen den Bedarfen der Institution und dem Kooperationspartner nicht recht gelingen mag.

Nina Simon inspiriert dazu, den Spagat zwischen der eigenen Institution und der Community auszuhalten, um an der Vision einer Bibliothek von (OF), durch (BY) und für die Community (FOR ALL) festzuhalten. Sie fordert uns dazu auf, die Bedarfe unserer Communities kennenzulernen und immer wieder Stellschrauben zu identifizieren, um diese wo möglich zu erfüllen. Das erfordert Zeit, viel Kommunikation und Vertrauensarbeit zwischen allen Beteiligten. Aber wir denken, es ist der Mühe wert.

\section{Zu Gast in der eigenen Bibliothek}

Abschließend möchten wir die wichtigsten Konsequenzen der Entscheidung für teilhabeorientierte Bibliotheksarbeit zusammenfassen. Auf der Ebene der Bibliothek als Institution werden Strukturen, Routinen, Ziele und Erwartungen herausgefordert und müssen selbstkritisch hinterfragt werden. Im Bereich Personalentwicklung geht es sowohl um einen Diversitäts-Ansatz im Recruiting als auch um Qualifizierung von „soft skills“. Außerdem geht es darum, intern eine partizipative Kultur aufzubauen mit der Konsequenz, dass es manchmal auch intern Unklarheiten in den Zielstellungen gibt und Konflikte entstehen, die moderiert werden müssen. Um auf der Ebene des Programm-Angebots den Anteil der Co-Produktionen und Schlüsselübergaben im Programm zu erhöhen, müssen wir als Institution die Perspektive wechseln und Verständigung zwischen oft sehr verschiedenen Arbeitsweisen und Betriebskulturen zulassen. Das erfordert Geduld, Toleranz und Kreativität. Schließlich ist teilhabeorientierte Bibliotheksarbeit eine Frage der Haltung: Sind wir bereit, in einem definierten Rahmen Entscheidungen und Deutungshoheit an die Besucher*innen abzugeben? Das bedeutet, den Mut aufzubringen, Berlin und seinen Communities immer öfter die Gastgeber*innen-Rolle zu überlassen und in unserer eigenen Bibliothek auch mal zu Gast zu sein.

\section{Literaturverzeichnis}

Borsky, Jenny; Hartung, Lea (2019): Informieren, Konsultieren, KoKreieren: Der Themenraum der ZLB als Werkstatt für Partizipation. In: BuB - Forum Bibliothek und Information, 71 (7), 426-27. Hartung, Lea; Lienhard, Claudia; Schütte, Sandra (2018): NutzerInnenpartizipation in Bibliotheken: Ergebnisse eines Hands-On
Labs. In: BuB - Forum Bibliothek und Information, 70 (10), 534-35.

Klinenberg, Eric (2018): Palaces for the people: How social infrastructure can help fight inequality, polarization, and the decline of civic life. New York: Crown.

Lankes, Richard David (2016): Expect more: Demanding better libraries for today's complex world. Syracuse, NY.

Lankes, Richard David (2018): Die andere Bibliothek - Brief an eine Altbekannte. In: Magazin der Kulturstiftung des Bundes, 30, 30-31. Verfügbar unter https://www.kulturstiftung-des-bundes. de/de/magazin/magazin_30/die_andere_bibliothek_brief_an_ eine_altbekannte.html.

Leik, Tim (2020): „Am Wochenende könnt ihr den Schlüssel haben!“ Die Sonntagsöffnung in der Amerika-Gedenkbibliothek. In: Bibliotheken als Orte kuratorischer Praxis, hg. v. von Klaus Ulrich Werner. Berlin: De Gruyter Saur, 203-14.

Lüttringhaus, Maria (2000): Stadtentwicklung und Partizipation. Fallstudien aus Essen Katernberg und der Dresdner Äußeren Neustadt. Bonn: Stiftung Mitarbeit.

ZLB (2018): Die Bibliothek der Zukunft. Verfügbar unter https://www. zlb.de/ueber-uns/positionen.

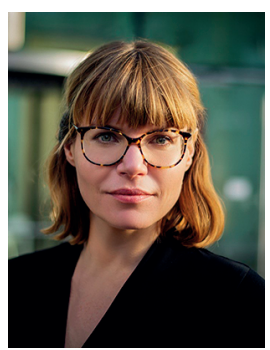

\section{Judith Galka}

Referentin des Vorstands / Generaldirektors

Zentral- und Landesbibliothek Berlin

Postfach 610179

D-10922 Berlin

judith.galka@zlb.de

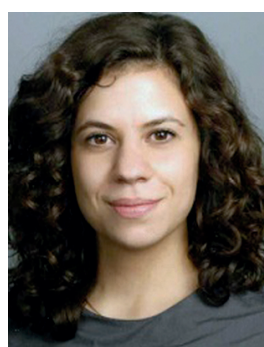

Jolina Haddad

Projektmitarbeiterin Community-Projekte Referat Programm

Abteilung Publikumsdienste

Zentral- und Landesbibliothek Berlin

Postfach 610179

D-10922 Berlin

jolina.haddad@zlb.de

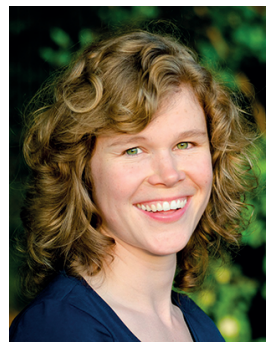

\section{Dr. Lea Hartung}

Projektleitung Community-Projekte

Referat Programm

Abteilung Publikumsdienste

Zentral- und Landesbibliothek Berlin

Postfach 610179

D-10922 Berlin

lea.hartung@zlb.de 